\title{
Long-term oncologic outcomes of radiotherapy combined with maximal androgen blockade for localized, high-risk prostate cancer
}

Yong Luo* (D), Mingchuan Li, Hengzhi Qi, Jiahui Zhao, Yili Han, Yunhua Lin, Zhu Hou and Yongguang Jiang*

\begin{abstract}
Background: To assess the oncologic outcomes of radiation therapy (RT) combined with maximal androgen blockade (MAB) and prostate-specific antigen (PSA) kinetics in patients with localized, high-risk prostate carcinoma (PCa).

Methods: Three-hundred twenty individuals with localized PCa who underwent RT + MAB in 2001-2015 were evaluated retrospectively. All patients had received 36 months of MAB therapy and 45 Gy of pelvic irradiation, plus a dose-escalated external beam radiation therapy (DE-EBRT) boost to 76 81 Gy (MAB + EBRT group), or a low-dose-rate prostate permanent brachytherapy (LDR-PPB) boost to 110 Gy with l-125 (MAB + EBRT + PPB group).

Results: Follow-up median is 90 months, ranging from 12 to 186 months; 117 (36.6\%) and 203 (63.4\%) cases underwent $M A B+E B R T$ and $M A B+E B R T+P P B$, respectively. Multivariate Cox regression showed that the $P P B$ regimen and PSA kinetics were positive indicators of oncologic outcomes. Compared with MAB + EBRT, MAB + EBRT + PPB remarkably improved PSA kinetics more pronouncedly: PSA nadir $(1.3 \pm 0.7 \mathrm{vs} 0.11 \pm 0.06 \mathrm{ng} / \mathrm{mL})$; time of PSA decrease to nadir (7.5 \pm 1.8 vs $3.2 \pm 2.1$ months); PSA doubling time (PSADT; $15.6 \pm 4.2$ vs $22.6 \pm 6.1$ months); decrease in PSA ( $84.6 \pm 6.2 \%$ vs $95.8 \pm 3.4 \%)$. Additionally, median times of several important oncologic events were prolonged in the MAB + EBRT + PPB group compared with the MAB + EBRT group: overall survival (OS; 12.3 vs 9.1 years, $P<0.001$ ), biochemical recurrence-free survival (BRFS; 9.8 vs 6.5 years, $P<0.001$ ), skeletal-related event (SRE; 10.4 vs 8.2 years, $P<0.001$ ), and cytotoxic chemotherapy (CCT; 11.6 vs 8.8 years, $P=0.007$ ).
\end{abstract}

Conclusion: $M A B+E B R T+P P B$ is extremely effective in patients with localized, high-risk PCa, indicating that PPB may play a synergistic role in improving PSA kinetics and independently predicts oncologic outcomes.

Keywords: Prostate cancer, Brachytherapy, PSA kinetics, Maximal androgen blockade, Radiation therapy

\section{Background}

Prostate cancer $(\mathrm{PCa})$ is a common malignancy characterized by both elevated morbidity and mortality. Most PCa patients, including those with high-risk disease, do not have metastatic tumors at diagnosis; therefore, local tumor resection could produce excellent long-term survival outcomes [1-3]. In individuals with localized, highrisk disease, not suitable for radical prostatectomy (RP),

\footnotetext{
* Correspondence: luoyonganzhen@163.com; jyganzhen@163.com Department of Urology, Beijing Anzhen Hospital, Capital Medical University, Anzhenli Street, Chaoyang District, Beijing 100029, People's Republic of China
}

combination therapies involving RT and androgen deprivation therapy (ADT) are preferred approaches.

As a new RT technique, modern brachytherapy was first applied for PCa in the 1980s when transrectal ultrasound became available to plan and guide radioactive seed placement within the prostate. Because of excellent 15-year PSA outcomes [4], brachytherapy is routinely performed either as monotherapy in individuals with low-risk or low-/intermediate-risk cancer or in combination with external beam radiation therapy (EBRT) in those with high-risk tumors [5]. A recent comprehensive literature review screening 18,000 articles with over

(C) The Author(s). 2018 Open Access This article is distributed under the terms of the Creative Commons Attribution 4.0 International License (http://creativecommons.org/licenses/by/4.0/), which permits unrestricted use, distribution, and 
50,000 patients comparatively analyzed PSA-free survival outcomes in patients suffering from localized PCa treated with different radical therapies [1]. The results suggested that PSA outcomes are significantly favorable after brachytherapy in comparison with EBRT in lowrisk cases, with the brachytherapy monotherapy achieving equivalent PSA outcomes compared to the EBRT and brachytherapy combination in individuals with intermediate-risk tumors. Here, we evaluated the clinical benefit of the MAB + EBRT + PPB combination by assessing long-term survival outcomes and PSA kinetics in subjects with localized high risk.

\section{Methods}

\section{Subjects}

All patients with localized, high-risk $\mathrm{PCa}$ treated by RT plus 36 months of MAB therapy from 01/01/2001 to $06 / 30 / 2015$ in our institution were enrolled in the present retrospective analysis. Some patients underwent the dose-escalated external beam radiation therapy (DE-EBRT) protocol of pelvic irradiation to $45 \mathrm{~Gy}$ and prostate irradiation to $76 \sim 81$ Gy (MAB + EBRT group), while the remaining cases were administered combined $\mathrm{RT}$ protocol of pelvic irradiation to 45 Gy and LDR-PPB to 110 Gy (MAB + EBRT + PPB group).

The patients were clinically diagnosed by determining serum PSA levels, transrectal prostate ultrasonography, pathological examination of puncture biopsy specimens or surgically removed samples, radioisotope scan of the bone, and abdominal and pelvic computed tomography. Follow-up for all patients ended on 06/ $30 / 2016$ in this retrospective clinical trial. Risk classification was based on the Memorial Sloan-Kettering group definition, in which patients are classified as having low (PSA $\leq 10 \mathrm{ng} / \mathrm{mL}$, Gleason score $\leq 6$, and clinical stage $\leq \mathrm{T} 2 \mathrm{a}$ ), intermediate (PSA $=10-20 \mathrm{ng} / \mathrm{mL}$, Gleason score $=7$, and/or clinical stage $\mathrm{T} 2 \mathrm{~b}$ ), and high (PSA $\geq 20 \mathrm{ng} / \mathrm{mL}$, Gleason score $\geq 8$, clinical stage $\geq \mathrm{T} 2 \mathrm{c}$, and/or two to three intermediate-risk features) risk.

\section{Patient follow-up and data collection}

Patients were monitored by serum PSA assessment quarterly for year 1 , then at 6-month intervals for year 2, and once a year afterwards. During follow-up, we measured PSA kinetics, including PSA nadir, the time required for PSA to reach nadir, and PSA decrease. In addition, PSA doubling time (PSADT) was determined as previously reported [6]. Furthermore, radioisotope scan of the bone and computed tomography of the pelvis, lung, and skull were performed every year.

\section{Study endpoints}

Primary study endpoints were OS (time elapsed from treatment to death) and BRFS (time to PSA biochemical recurrence). Secondary endpoints included SRE-free survival (SRE-FS; time to the first SRE) and CCT-free survival (CCT-FS; time to the first CCT). PSA kinetics was also assessed as described above. PSA biochemical recurrence was reflected by more than 1.25 -fold elevation compared to baseline values (for cases with no previous PSA level decrease) or exceeding the nadir level (for the remaining cases), and absolute PSA amounts increased by $\geq 2 \mathrm{ng} / \mathrm{mL}$ [7]. Radiotherapy or bone surgery, pathologic bone fractures, spinal cord compression, and antineoplastic treatment changes for bone pain alleviation were generally considered SRE.

\section{Adverse effect assessment}

In the form of telephone inquiring and questionnaire, we regularly monitored the complications of patients during the treatment process. Acute symptoms were related to radiation effects on proliferating tissues at the time of radiation treatment, and late symptoms occurred months after radiation treatment and were likely to remain. Additionally, we also observed the possible complications related to ADT on multiple system functions, such as endocrine symptoms, sexual function, cardiovascular events, and several important organ functions. Acute urogenital symptoms were classified according to the standard recommended by American Brachytherapy Society (ABS) as the following: grade 0 , without any complication; grade 1, mild urination burning and frequency (2-3 times every night), no intervention required; grade 2 , moderate urination burning and frequency (4-6 times every night) and gross hematuria, but conservative measures are generally effective; grade 3 , severe urination burning and frequency (7-10 times every night) and gross hematuria, requiring active intervention; grade 4, severe hesitancy or retention, requiring catheterization. Acute rectal symptoms were evaluated using Radiation Therapy Oncology Group (RTOG) toxicity scoring criteria: grade 0 , without any complication; grade 1 , symptoms of rectal frequency, urgency, tenesmus or mucoid stool, which need to be treated with conservative measures; grade 2 , intermittent rectal bleeding, rectum erythema, requiring active intervention; grade 3, rectal ulceration and severe bleeding, which would require emergent colonoscopy fulguration and blood transfusion; grade 4, intestinal obstruction or fistula, massive rectal bleeding, which need to be emergently treated with surgery or vascular support.

\section{Statistical analysis}

Prognostic parameters were first evaluated by univariate (log-rank) and multivariate (Cox regression) analyses. 
Next, PSA kinetics was compared between the two treatment groups by independent sample $t$ test. Furthermore, OS, BRFS, SRE-FS, and CCT-FS curves were obtained by the Kaplan-Meier method. To test the statistical significance of the difference in adverse effects between the two groups, chi-square test was done. $P<0.05$ was considered to reflect statistical significance.

\section{Results}

\section{Patients' characteristics}

In this study, 320 subjects with localized, high-risk $\mathrm{PCa}$ administered combination treatment of RT + MAB were included. Median follow-up was 90 months (12 186 months). Among the patients, 117 (36.6\%) cases underwent MAB + EBRT and 203 (63.4\%) received $\mathrm{MAB}+\mathrm{EBRT}+\mathrm{PPB}$. The detailed clinical and treatment characteristics of the patients are provided in Table 1.

\section{Factors influencing survival prognosis}

Table 2 summarizes univariate and multivariate analyses for OS predictors. Univariate analysis indicated that age $(P=0.035$, hazard ratio [HR] 5.812), gland volume $(P=0.006$, HR 3.816$)$, PPB addition $(P=0.007$, HR 3.016$)$, clinical stage $(P<0.001$, HR 4.557), Gleason score $(P=$ 0.001, HR 3.356), baseline PSA $(P=0.027$, HR 1.558), Memorial Sloan-Kettering Risk Classification Standard score $(P<0.001$, HR 7.658$)$, PSA nadir $(P<0.001$, HR 9 . $473)$, time to PSA nadir ( $P=0.012$, HR 3.113), PSADT $(P=0.042$, HR 2.665$)$, and PSA level reduction $(P<0.001$, HR 13.463) were significant predictors of OS in patients with localized, high-risk PCa. Multivariate Cox regression analysis further identified gland volume $(P=0.042$, HR 1.192), PPB addition $(P<0.001$, HR 6.358), clinical stage $(P=0.011$, HR 2.183$)$, Gleason score $(P<0.001$, HR 7.142), baseline PSA $(P=0.014$, HR 3.492), Memorial Sloan-Kettering Risk Classification Standard score $(P<0.001$, HR 5.479$)$, PSA nadir $(P=0.012$, HR 4.553), time to PSA nadir ( $P=0.038$, HR 1.249$)$, PSADT $(P=0.028$, HR 5.511), and PSA reduction $(P<0.001$, HR $7.845)$ as independent prognostic indicators of OS

The prognostic indicators of BRFS are presented in Table 3. Univariate analysis indicated that gland volume $(P=0.047$, HR 3.668), MAB pattern $(P=0.031$, HR 1.492), PPB addition $(P=0.001$, HR 2.888$)$, clinical stage $(P=$ 0.041, HR 4.737), Gleason score $(P=0.013$, HR 5.711), baseline PSA $(P=0.019$, HR 2.622), Memorial SloanKettering Risk Classification Standard score $(P=0.027$, HR 1.772), PSA nadir $(P=0.041$, HR 1.323$)$, time to PSA nadir $(P=0.032$, HR 2.116$)$, PSADT $(P=0.048$, HR 1.863), and PSA reduction $(P=0.006$, HR 3.677) were significant predictors of BRFS in patients with localized, high-risk PCa. Multivariate Cox regression analysis further identified gland volume $(P=0.016$,
Table 1 Clinical and treatment characteristics of the patients

\begin{tabular}{|c|c|c|}
\hline & Median & Range \\
\hline Age at diagnosis (years) & 70 & $58 \sim 81$ \\
\hline Gland volume (mL) & 33.4 & $27 \sim 62$ \\
\hline \multirow[t]{2}{*}{ Follow-up (months) } & 90 & $12 \sim 186$ \\
\hline & Count & Percentage (\%) \\
\hline \multicolumn{3}{|l|}{ Clinical stage } \\
\hline $\mathrm{T} 2 \mathrm{~b}$ & 23 & 7.2 \\
\hline $\mathrm{T} 2 \mathrm{C}$ & 167 & 52.2 \\
\hline T3a & 82 & 25.6 \\
\hline $\mathrm{T} 3 \mathrm{~b}$ & 48 & 15 \\
\hline \multicolumn{3}{|l|}{ Gleason score } \\
\hline$\leq 6$ & 3 & 0.9 \\
\hline 7 & 11 & 3.4 \\
\hline$\geq 8$ & 306 & 95.6 \\
\hline \multicolumn{3}{|l|}{ PSA at diagnosis (ng/mL) } \\
\hline$\leq 10$ & 35 & 10.9 \\
\hline $10 \sim 20$ & 49 & 15.3 \\
\hline$\geq 20$ & 236 & 73.8 \\
\hline \multicolumn{3}{|c|}{ Memorial Sloan-Kettering risk classification } \\
\hline $2 \sim 3 \mathrm{IS}$ & 71 & 22.2 \\
\hline $1 \mathrm{HS}$ & 106 & 33.1 \\
\hline 2 3 HS & 143 & 44.7 \\
\hline \multicolumn{3}{|l|}{$M A B$} \\
\hline Continuous & 184 & 57.5 \\
\hline Intermittent & 136 & 42.5 \\
\hline \multicolumn{3}{|l|}{ PPB } \\
\hline Yes & 203 & 63.4 \\
\hline No & 117 & 36.6 \\
\hline \multicolumn{3}{|l|}{ PSA nadir (ng/mL) } \\
\hline$\leq 1.0$ & 241 & 75.3 \\
\hline$>1.0$ & 79 & 24.7 \\
\hline \multicolumn{3}{|c|}{ Time to PSA nadir (months) } \\
\hline$\leq 3$ & 207 & 64.7 \\
\hline$>3$ & 113 & 35.3 \\
\hline \multicolumn{3}{|c|}{ PSA doubling time (months) } \\
\hline$\leq 12$ & 46 & 14.4 \\
\hline$>12$ & 274 & 85.6 \\
\hline \multicolumn{3}{|l|}{ PSA decrease (\%) } \\
\hline$<90$ & 71 & 22.2 \\
\hline$\geq 90$ & 249 & 77.8 \\
\hline
\end{tabular}

MAB Maximal Androgen Blockade; PPB Permanent Prostate Brachytherapy; IS intermediate-Risk Standard; HS High-Risk Standard

HR 8.336), MAB pattern $(P=0.018$, HR 3.217), PPB addition $(P<0.001$, HR 5.126), clinical stage $(P=0.013$, HR 6.142), Gleason score $(P=0.022$, HR 3.463), PSA 
Table 2 Analyses for prognostic indicators of Overall Survival

\begin{tabular}{|c|c|c|c|c|}
\hline \multirow[t]{3}{*}{ Variable } & \multicolumn{4}{|c|}{ Overall Survival } \\
\hline & \multicolumn{2}{|c|}{ Univariate analysis } & \multicolumn{2}{|c|}{ Multivariate analysis } \\
\hline & P Value & $\mathrm{HR}$ & P Value & $H R$ \\
\hline $\begin{array}{l}\text { Age at diagnosis (years) } \\
\leq 70 \text { vs }>70\end{array}$ & 0.035 & 5.812 & 0.174 & - - \\
\hline $\begin{array}{l}\text { Gland volume }(\mathrm{mL}) \\
\leq 33 \text { vs }>33\end{array}$ & 0.006 & 3.816 & 0.042 & 1.192 \\
\hline $\begin{array}{l}\text { MAB } \\
\text { Continuous vs Intermittent }\end{array}$ & 0.583 & - & 0.457 & - \\
\hline $\begin{array}{l}\text { PPB } \\
\text { yes vs no }\end{array}$ & 0.007 & 3.016 & $<0.001$ & 6.358 \\
\hline $\begin{array}{l}\text { Clinical stage } \\
\leq \mathrm{T} 2 \mathrm{c} \text { vs } \geq \mathrm{T} 3 \mathrm{a}\end{array}$ & $<0.001$ & 4.557 & 0.011 & 2.183 \\
\hline $\begin{array}{l}\text { Gleason score } \\
\leq 7 \text { vs } \geq 8\end{array}$ & 0.001 & 3.356 & $<0.001$ & 7.142 \\
\hline $\begin{array}{l}\text { PSA at diagnosis (ng/mL) } \\
\quad \leq 10 \text { vs } 10 \sim 20 \text { vs } \geq 20\end{array}$ & 0.027 & 1.558 & 0.014 & 3.492 \\
\hline $\begin{array}{l}\text { Memorial Sloan-Kettering risk classification } \\
2 \sim 3 \text { IS vs } 1 \text { HS vs } 2 \text { HS }\end{array}$ & $<0.001$ & 7.658 & $<0.001$ & 5.479 \\
\hline $\begin{array}{l}\text { PSA nadir (ng/mL) } \\
\quad \leq 1 \text { vs }>1\end{array}$ & $<0.001$ & 9.473 & 0.012 & 4.553 \\
\hline $\begin{array}{l}\text { Time to PSA nadir (months) } \\
\leq 3 \text { vs }>3\end{array}$ & 0.012 & 3.113 & 0.038 & 1.249 \\
\hline $\begin{array}{l}\text { PSA doubling time (months) } \\
\leq 12 \text { vs }>12\end{array}$ & 0.042 & 2.665 & 0.028 & 5.511 \\
\hline $\begin{array}{l}\text { PSA decrease (\%) } \\
<90 \text { vs } \geq 90\end{array}$ & $<0.001$ & 13.463 & $<0.001$ & 7.845 \\
\hline
\end{tabular}

MAB Maximal Androgen Blockade; PPB Permanent Prostate Brachytherapy; IS intermediate-Risk Standard; HS High-Risk Standard

baseline $(P=0.001$, HR 6.334), Memorial Sloan-Kettering Risk Classification Standard score $(P=0.009$, HR 3.643), PSA nadir $(P=0.016$, HR 6.993), time to PSA nadir $(P=0.011$, HR 5.843), PSADT $(P=0.014$, HR 6.132), and PSA reduction $(P<0.001$, HR 9.385) as independent prognostic indicators of BRFS.

\section{Characteristics of high-risk patients treated with different $\mathrm{RT}$ regimens}

To assess how different RT regimens affect the PSA kinetics and oncologic outcomes, we further divided the high-risk patients into two different treatment groups, whose clinical and pathological characteristics are summarized in Table 4.

\section{PSA kinetics in high-risk patients treated with different RT regimens}

As shown in Fig. 1, the parameters of PSA kinetics were affected by different RT regimens in patients with localized, high-risk PCa. PSA nadir values in the MAB + EBRT and $\mathrm{MAB}+\mathrm{EBRT}+\mathrm{PPB}$ combination groups were $1.3 \pm 0.7 \mathrm{ng} / \mathrm{mL}$ (range, $0.03-14.5 \mathrm{ng} / \mathrm{mL}$ ) and 0.11 $\pm 0.06 \mathrm{ng} / \mathrm{mL}$ (range, $0.00-1.27 \mathrm{ng} / \mathrm{mL}$ ), respectively. Times of PSA decrease to nadir in these two groups were $7.5 \pm 1.8$ months (range, $3-12$ months) and $3.2 \pm 2.1$ months (range, 1-9 months), respectively. Meanwhile, PSADT values were $15.6 \pm 4.2$ months (range, 7.1-27.4 months) in the MAB + EBRT group and 22.6 \pm 6.1 months (range, 7.6-43.2 months) in the MAB + EBRT + PPB group. Finally, PSA levels were reduced by $84.6 \pm 6.2 \%$ (range, 67.1-94.5\%) in the MAB + EBRT group and $95.8 \pm 3.4 \%$ (range, 83.1-99.99\%) in the MAB + EBRT + PPB group. These findings demonstrated that PSA kinetics, which is an important independent indicator of OS, and BRFS could be notably improved by PPB-based combination regimens.

\section{Endpoint events in high-risk patients treated with different $\mathrm{RT}$ regimens}

As shown in Fig. 2a, the 5-, 7-, 10-, 12-, and 15-year overall survival rates in the $\mathrm{MAB}+\mathrm{EBRT}+\mathrm{PPB}$ group were markedly higher than those in the MAB + EBRT group (99.4 vs $96.6 \%, P=0.241$; 98.3 vs $93.4 \%, P=0.039$; 97.2 vs $87.3 \%, P=0.011 ; 94.5$ vs $81.8 \%, P=0.003 ; 91.4$ vs $76.5 \%, P<0.001)$. Median OS was 9.1 years [95\% confidence interval (CI) 7.5 to 12.6 ] among patients receiving $\mathrm{MAB}+$ EBRT and 12.3 years (95\% CI 10.6 to 13.2$)$ in those administered MAB + EBRT + PPB (HR 6.358, 95\% CI 5.733 to $6.627, P<0.001)$. Meanwhile, the PPB-based combination regimen significantly increased the median 
Table 3 Analyses for prognostic indicators of Biochemical Recurrence-Free Survival

\begin{tabular}{|c|c|c|c|c|}
\hline \multirow[t]{3}{*}{ Variable } & \multicolumn{4}{|c|}{ Biochemical Recurrence-Free Survival } \\
\hline & \multicolumn{2}{|c|}{ Univariate analysis } & \multicolumn{2}{|c|}{ Multivariate analysis } \\
\hline & P Value & $H R$ & P Value & $H R$ \\
\hline $\begin{array}{l}\text { Age at diagnosis (years) } \\
\leq 70 \text { vs }>70\end{array}$ & 0.339 & - & 0.147 & -— \\
\hline $\begin{array}{l}\text { Gland volume }(\mathrm{mL}) \\
\leq 33 \text { vs }>33\end{array}$ & 0.047 & 3.668 & 0.016 & 8.336 \\
\hline $\begin{array}{l}\text { MAB } \\
\text { Continuous vs Intermittent }\end{array}$ & 0.031 & 1.492 & 0.018 & 3.217 \\
\hline $\begin{array}{l}\text { PPB } \\
\text { yes vs no }\end{array}$ & 0.001 & 2.888 & $<0.001$ & 5.126 \\
\hline $\begin{array}{l}\text { Clinical stage } \\
\leq \mathrm{T} 2 \mathrm{c} \text { vs } \geq \mathrm{T} 3 \mathrm{a}\end{array}$ & 0.041 & 4.737 & 0.013 & 6.142 \\
\hline $\begin{array}{l}\text { Gleason score } \\
\leq 7 \text { vs } \geq 8\end{array}$ & 0.013 & 5.711 & 0.022 & 3.463 \\
\hline $\begin{array}{l}\text { PSA at diagnosis ( } \mathrm{ng} / \mathrm{mL}) \\
\leq 10 \text { vs } 10 \sim 20 \mathrm{vs} \geq 20\end{array}$ & 0.019 & 2.622 & 0.001 & 6.334 \\
\hline $\begin{array}{l}\text { Memorial Sloan-Kettering risk classification } \\
2 \sim 3 \text { IS vs } 1 \text { HS vs } 2 \text { HS }\end{array}$ & 0.027 & 1.772 & 0.009 & 3.643 \\
\hline $\begin{array}{l}\text { PSA nadir }(\mathrm{ng} / \mathrm{mL}) \\
\leq 1 \text { vs }>1\end{array}$ & 0.041 & 1.323 & 0.016 & 6.993 \\
\hline $\begin{array}{l}\text { Time to PSA nadir (months) } \\
\leq 3 \text { vs }>3\end{array}$ & 0.032 & 2.116 & 0.011 & 5.843 \\
\hline $\begin{array}{l}\text { PSA doubling time (months) } \\
\leq 12 \text { vs }>12\end{array}$ & 0.048 & 1.863 & 0.014 & 6.132 \\
\hline $\begin{array}{l}\text { PSA decrease (\%) } \\
\quad<90 \text { vs } \geq 90\end{array}$ & 0.006 & 3.677 & $<0.001$ & 9.385 \\
\hline
\end{tabular}

MAB Maximal Androgen Blockade; PPB Permanent Prostate Brachytherapy; IS intermediate-Risk Standard; HS High-Risk Standard

time of PSA biochemical progression, from 6.5 years (95\% CI 4.8 to 8.1 ) in the MAB + EBRT group to 9.8 years (95\% CI 8.5 to 10.7) in MAB + EBRT + PPB-treated patients (HR 5.126, 95\% CI 4.251 to $6.306, P<0.001$ )

(Fig. 2b).

A median time to first SRE of 10.4 years (95\% CI 8.9 to 12.2) was found in the MAB + EBRT + PPB group, compared with 8.2 years (95\% CI 7.1 to 10.5 ) in $\mathrm{MAB}+$ EBRT-treated individuals, indicating significantly reduced risk of SRE (HR 3.361, 95\% CI 2.925 to 3.815, $P<0.001$ ) (Fig. 3a). The superiority of MAB + EBRT + PPB over $\mathrm{MAB}+$ EBRT was also shown for CCT initiation (Fig. 3b). Indeed, median times to CCT initiation were 11.6 years (95\% CI 9.8 to 12.7 ) and 8.8 years (95\% CI 6.3 to 10.9 in the $\mathrm{MAB}+\mathrm{EBRT}+\mathrm{PPB}$ and $\mathrm{MAB}+\mathrm{EBRT}$ groups, respectively. Treatment with the PPB-based combination regimen could remarkably increase the CCT-FS rate compared with MAB + EBRT (HR 1.627, 95\% CI 1.311 to 1.809, $P=0.007)$.

\section{Complications in high-risk patients treated with different $\mathrm{RT}$ regimens}

As shown in Table 5, the complication rates between $\mathrm{MAB}+\mathrm{EBRT}$ group and $\mathrm{MAB}+\mathrm{EBRT}+\mathrm{PPB}$ group showed no significant differences in late radiationrelated symptoms and multiple organ functions.

Although the group of MAB + EBRT + PPB patients displayed significant higher complications rates than those of MAB + EBRT cases in grade 2 (31.53 vs 20.51\%, $P=0.034)$ and grade $3(23.15$ vs $5.13 \%, P<0.001)$ of acute urogenital symptoms, all these symptoms could be improved gradually. Fifteen patients (12.82\%) in MAB + EBRT group and 30 patients (14.78\%) in $\mathrm{MAB}+\mathrm{EBRT}+\mathrm{PPB}$ group were identified as ABS grade 4 because of retention and catheterization, and catheter could be removed in the vast majority of these cases successfully. One patient $(0.85 \%)$ in $\mathrm{MAB}$ + EBRT group and four patients (1.97\%) in MAB + EBRT + PPB group were diagnosed as RTOG grade 3 due to rectal ulceration and severe bleeding, which were successfully treated with colonoscopy fulguration. And none of all patients developed to symptoms of RTOG grade 4. Additionally, only one case in the group of MAB + EBRT + PPB developed to intestinal fistula and received repair surgery.

In all, the combination therapy of MAB + EBRT + PPB showed similar safety to MAB + EBRT regimen, and no significant serious complications were observed in $\mathrm{MAB}+\mathrm{EBRT}+\mathrm{PPB}$ regimen. 
Table 4 Comparison of the characteristics of high-risk patients undergone different treatment

\begin{tabular}{|c|c|c|c|}
\hline \multirow[t]{2}{*}{ Treatment } & $\begin{array}{l}\text { MAB+EBRT } \\
(n=117)\end{array}$ & $\begin{array}{l}\text { MAB+EBRT+PPB } \\
(n=203)\end{array}$ & \multirow[b]{2}{*}{ P Value } \\
\hline & Median (Range) & Median (Range) & \\
\hline Age at diagnosis (years) & $70(59 \sim 81)$ & 69 (58 79) & 0.11 \\
\hline Gland volume (mL) & $32.3(27 \sim 62)$ & $34.6(29 \sim 62)$ & 0.09 \\
\hline \multirow[t]{2}{*}{ Follow-up (months) } & $84(12 \sim 186)$ & $90(12 \sim 186)$ & 0.25 \\
\hline & Count (\%) & Count (\%) & P Value \\
\hline \multicolumn{4}{|l|}{ Clinical stage } \\
\hline $\mathrm{T} 2 \mathrm{~b}$ & $9(7.7)$ & $14(6.9)$ & \multirow[t]{4}{*}{0.32} \\
\hline $\mathrm{T} 2 \mathrm{C}$ & $63(53.8)$ & $104(51.2)$ & \\
\hline Т3а & $28(23.9)$ & $54(26.6)$ & \\
\hline $\mathrm{T} 3 \mathrm{~b}$ & $17(14.5)$ & $31(15.3)$ & \\
\hline \multicolumn{4}{|l|}{ Gleason score } \\
\hline$\leq 6$ & $2(1.7)$ & $1(0.5)$ & \multirow[t]{3}{*}{0.27} \\
\hline 7 & $3(2.6)$ & $8(3.9)$ & \\
\hline$\geq 8$ & $112(95.7)$ & $194(95.6)$ & \\
\hline \multicolumn{4}{|l|}{ PSA at diagnosis (ng/mL) } \\
\hline$\leq 10$ & $17(14.5)$ & $18(8.9)$ & \multirow[t]{3}{*}{0.13} \\
\hline $10 \sim 20$ & $19(16.2)$ & $30(14.8)$ & \\
\hline$\geq 20$ & $81(69.2)$ & $155(76.4)$ & \\
\hline \multicolumn{4}{|c|}{ Memorial Sloan-Kettering risk classification } \\
\hline $2 \sim 3 \mathrm{IS}$ & $27(23.1)$ & $44(21.7)$ & \multirow[t]{3}{*}{0.61} \\
\hline $1 \mathrm{HS}$ & $38(32.5)$ & $68(33.5)$ & \\
\hline 2 3 HS & $52(44.4)$ & $91(44.8)$ & \\
\hline \multicolumn{4}{|l|}{ MAB } \\
\hline Continuous & $58(49.6)$ & $126(62.1)$ & \multirow[t]{2}{*}{0.07} \\
\hline Intermittent & $59(50.4)$ & $77(37.9)$ & \\
\hline
\end{tabular}

MAB Maximal Androgen Blockade; PPB Permanent Prostate Brachytherapy; IS Intermediate-Risk Standard; HS High-Risk Standard

\section{Discussion}

In the past 20 years, interstitial radiation therapy has been used as routine treatment for patients with clinically localized PCa, and most researchers believe that the 5-year PSA outcome of brachytherapy in low-risk patients is not statistically different from that of RP or EBRT. In addition, intermediate- and high-risk patients administered RP or EBRT may show a better response compared with those that undergo brachytherapy [8]. However, this view remains controversial. Polascik et al. reported that 7-year actuarial PSA progression-free survival following RP is remarkably higher than that of the I-125 brachytherapy group (97.8 vs $79 \%)$ [9, 10] in patients with localized PCa. Therefore, Polascik et al. proposed that brachytherapy should be cautiously recommended to patients with localized PCa. Sharkey and colleagues analyzed $1707 \mathrm{PCa}$ patients with T1 or T2 stage disease treated by either brachytherapy or RP; they concluded that the time to PSA-indicated recurrence is better controlled by brachytherapy than RP in intermediate- (89 vs 58\%, $P<0.05$ ) and high-risk ( 88 vs $43 \%, P<0$. 05) groups, but not in low-risk patients ( 89 vs $94 \%, P=$ 0.174) [11]. Moreover, Taira et al. evaluated 329 cases of high-risk PCa treated with brachytherapy + EBRT with a 10-year follow-up and found that cause-specific survival (CSS) in Gleason 5 patients is significantly lower than that of non-Gleason 5 patients $(90.3$ vs $98.1 \%, P=0.011)$. However, no remarkable differences in BRFS and OS between these two groups of patients were observed [12]. In addition, Demanes et al. retrospectively assessed 209 cases treated with brachytherapy + EBRT with a 10-year followup and reported OS and CSS rates of 79 and 97\%, respectively. Meanwhile, PSA progression-free survival rates were different for patients with low-, intermediate-, and highrisk disease (90, 87, and 69\%, respectively) [13]. Another study reported that the combination strategy of brachytherapy + EBRT is significantly more advantageous than brachytherapy monotherapy in 5-year biochemical 

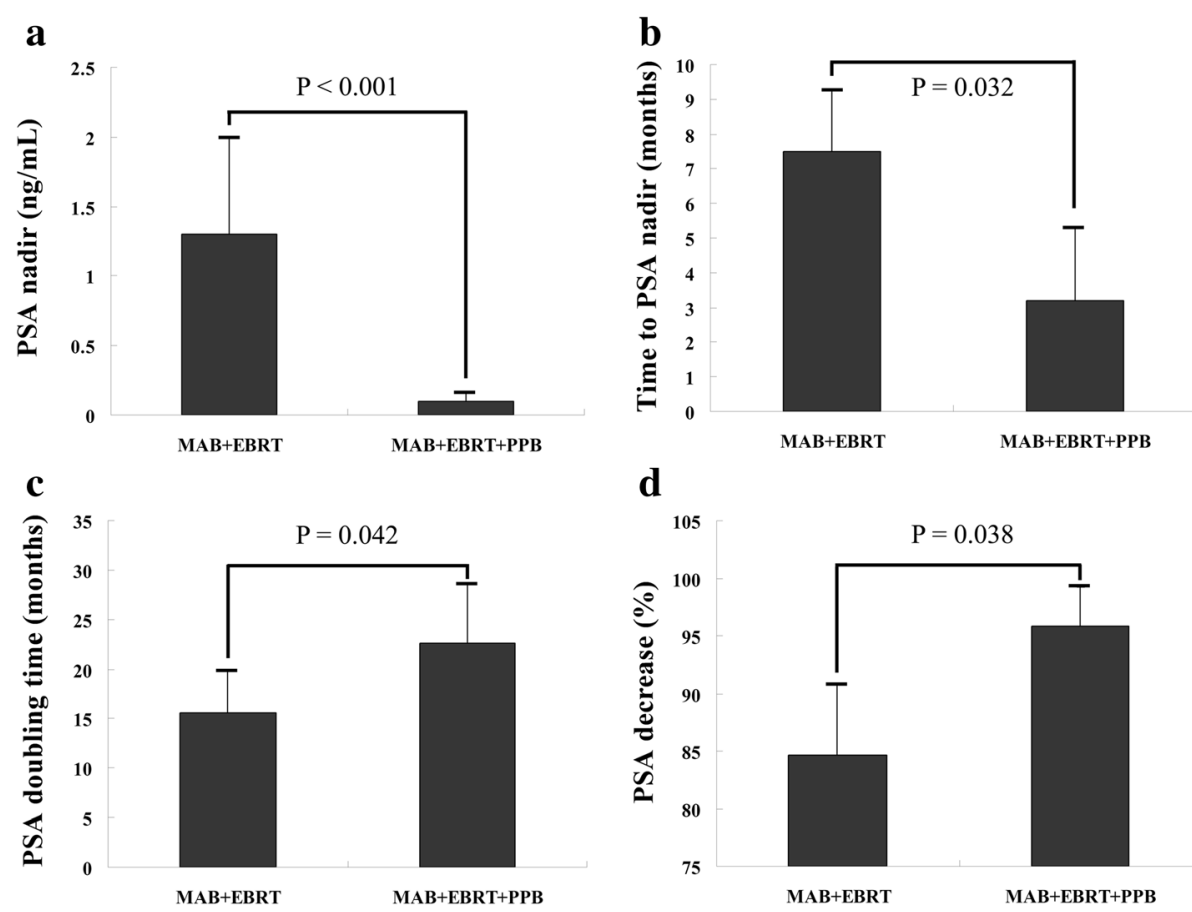

Fig. 1 Differences in PSA kinetics between cases treated by MAB + EBRT and MAB + EBRT + PPB combination therapies. a PSA nadir. $\mathbf{b}$ Time to PSA nadir. c PSA doubling time. $\mathbf{d}$ Declining extent of PSA

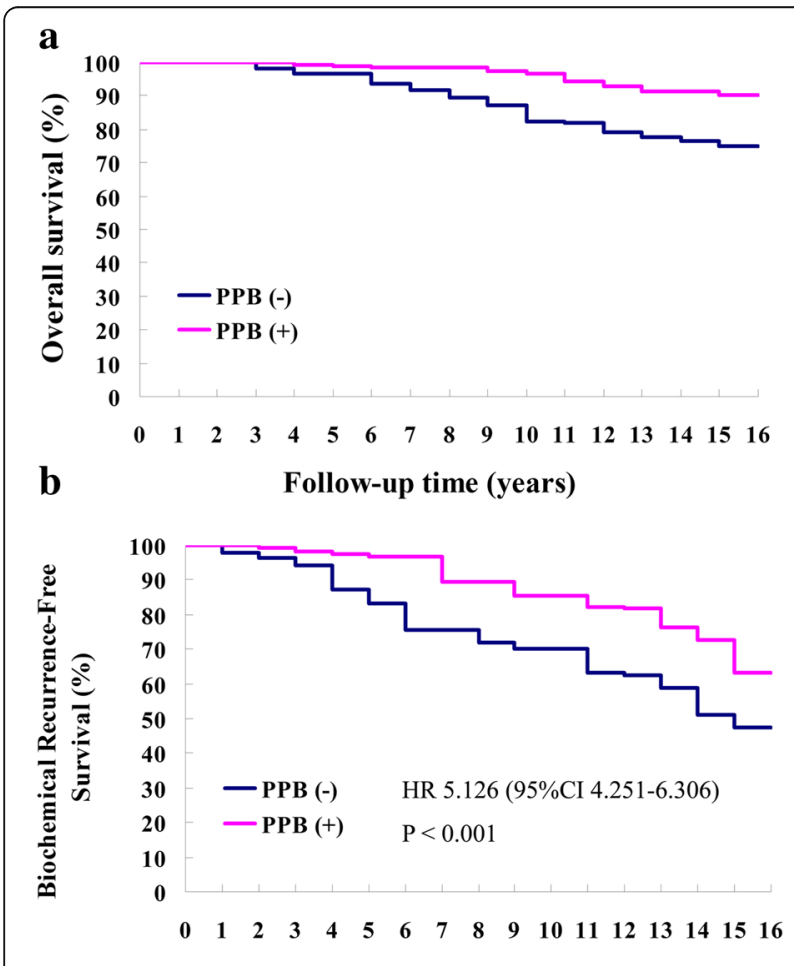

Follow-up time (years)

Fig. 2 a Overall survival of cases administered $M A B+E B R T$ and $M A B$ + EBRT + PPB combination therapies. b PSA biochemical recurrencefree survival of cases administered $M A B+E B R T$ and $M A B+E B R T+P P B$ combination therapies
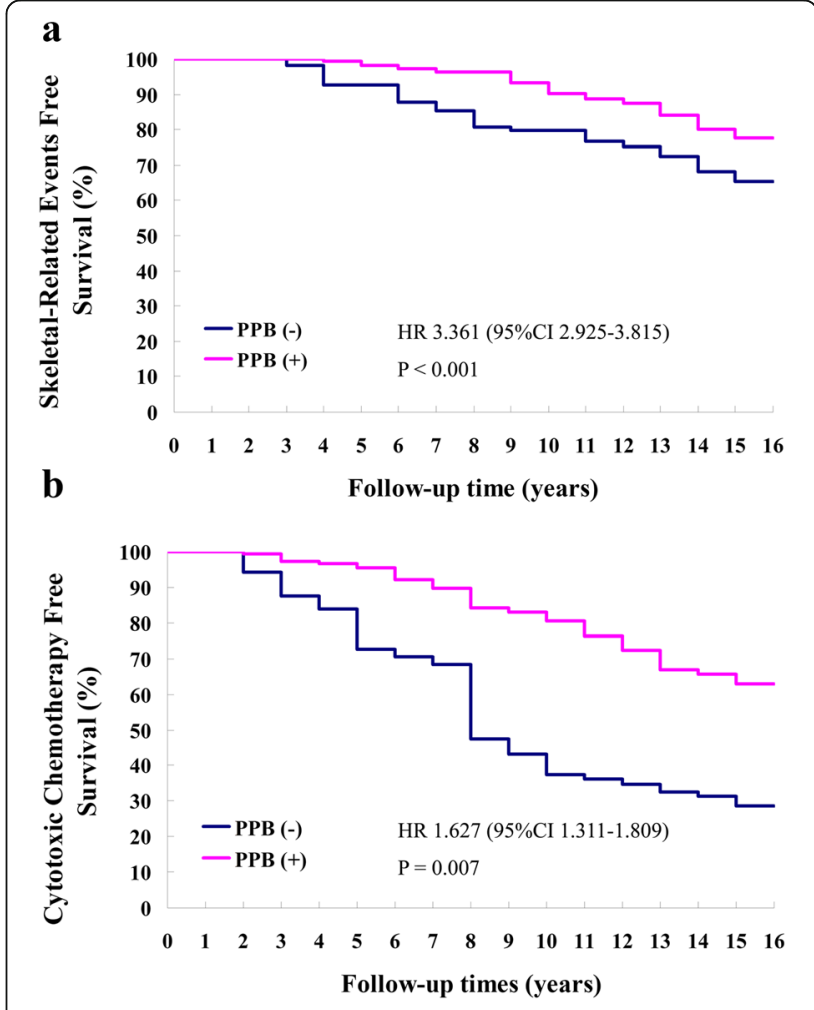

Fig. 3 a Skeletal-related event-free survival of cases administered MAB + EBRT and MAB + EBRT + PPB combination therapies. $\mathbf{b}$ Cytotoxic chemotherapy-free survival of cases administered MAB + EBRT and $M A B+E B R T+P P B$ combination therapies 
Table 5 Complications of high-risk patients undergone different treatment

\begin{tabular}{|c|c|c|c|c|}
\hline Adverse Effects & $\begin{array}{l}\text { MAB+EBRT } \\
\%(n=117)\end{array}$ & $\begin{array}{l}\text { MAB+EBRT+PPB } \\
\%(n=203)\end{array}$ & $x^{2}$ & P Value \\
\hline \multicolumn{5}{|l|}{ Acute Urology Function } \\
\hline ABS Grade 0 & 12.82 & 5.42 & 5.447 & 0.020 \\
\hline ABS Grade 1 & 48.72 & 25.12 & 18.480 & 0.000 \\
\hline ABS Grade 2 & 20.51 & 31.53 & 4.516 & 0.034 \\
\hline ABS Grade 3 & 5.13 & 23.15 & 17.449 & 0.000 \\
\hline ABS Grade 4 & 12.82 & 14.78 & 0.235 & 0.628 \\
\hline \multicolumn{5}{|l|}{ Late Urology Function } \\
\hline Urgent/Incontinence & 1.71 & 1.48 & 0.026 & 0.872 \\
\hline Hesitancy/Retention & 1.71 & 3.45 & 0.821 & 0.365 \\
\hline Gross Hematuria & 4.27 & 6.4 & 0.635 & 0.426 \\
\hline Stricture & 0 & 0.49 & 0.578 & 0.447 \\
\hline Frequency/Nocturia & 15.38 & 25.12 & 4.162 & 0.041 \\
\hline \multicolumn{5}{|l|}{ Acute Gastrointestinal Function } \\
\hline RTOG Grade 0 & 90.6 & 88.67 & 0.291 & 0.590 \\
\hline RTOG Grade 1 & 5.13 & 5.91 & 0.086 & 0.769 \\
\hline RTOG Grade 2 & 3.42 & 3.45 & 0.000 & 1.000 \\
\hline RTOG Grade 3 & 0.85 & 1.97 & 0.601 & 0.438 \\
\hline RTOG Grade 4 & 0 & 0 & - & - \\
\hline \multicolumn{5}{|l|}{ Late Gastrointestinal Function } \\
\hline Diarrhoea & 21.37 & 13.3 & 3.549 & 0.060 \\
\hline NauseaNomiting & 7.69 & 4.93 & 1.017 & 0.313 \\
\hline Abdominal Pain & 4.27 & 3.94 & 0.021 & 0.885 \\
\hline Rectal Bleeding & 5.13 & 10.34 & 2.614 & 0.106 \\
\hline Intestinal Fistula & 0 & 0.49 & 0.578 & 0.447 \\
\hline \multicolumn{5}{|l|}{ Endocriology Function } \\
\hline Breast Pain & 69.23 & 63.05 & 1.250 & 0.264 \\
\hline Xerosis Cutis & 11.11 & 19.7 & 3.966 & 0.046 \\
\hline Hot Flush & 20.51 & 37.44 & 9.897 & 0.002 \\
\hline Sexual Dysfunction & 53.85 & 47.78 & 1.091 & 0.296 \\
\hline \multicolumn{5}{|l|}{ Other System Function } \\
\hline Liver Dysfunction & 5.13 & 8.37 & 1.172 & 0.279 \\
\hline Renal Dysfunction & 7.69 & 6.4 & 0.192 & 0.661 \\
\hline Angina Pectoris & 34.18 & 25.12 & 2.997 & 0.083 \\
\hline Heart Failure & 7.69 & 2.96 & 3.727 & 0.054 \\
\hline Dyspnea & 3.42 & 0.99 & 2.389 & 0.122 \\
\hline Anaemia(moderate/severe) & 16.24 & 16.75 & 0.014 & 0.906 \\
\hline
\end{tabular}

Note: ABS American Brachytherapy Society, RTOG Radiation Therapy Oncology Group

relapse-free survival ( 80 vs $59 \%, P<0.01$ ), although EBRTtreated cases showed more adverse disease factors [14]. Collectively, current clinical evidence supports brachytherapy + EBRT as a proven treatment regimen for all stages of localized PCa [15].

In the present analysis, EBRT + MAB + PPB showed a significant benefit for long-term OS and BRFS in localized high-risk patients compared to EBRT + MAB combination. In addition, the brachytherapy-based combination treatment also postponed several important clinical events: 3.3 years for PSA biochemical recurrence, 2.2 years for SRE, and 2.8 years for CCT. The recently open-published ASCENDE-RT trial [16] compared survival endpoints between the DE-EBRT and low-dose-rate 
brachytherapy (LDR-BT) arms in intermediate-/high-risk patients and showed that DE-EBRT is twice as likely to result in biochemical failure, while OS rates were similar between these two treatment arms $(P=0.62)$.

Recent studies reported that PSA kinetics is closely related to long-term survival outcomes in PCa patients $[17,18]$. More importantly, PSA kinetics was confirmed to independently predict OS and BRFS by multivariate analysis in the current analysis. Specifically, PSA reduction over $90 \%$ was strongly associated with improved long-term survival as well as PSA biochemical progression in high-risk disease cases treated with RT + MAB. It is known that a short PSADT is associated with a promptly expanding tumor, a higher metastatic potential, and a somewhat elevated risk of cancer specific mortality [19-21]. D'Amico et al. found that patients with a PSADT less than 3 months represent $10-15 \%$ of males showing biochemical recurrence, but a higher risk of systemic recurrence $[20,21]$ and cancer-specific mortality with a median survival of 6 years [22]. Similarly, in men post-RT, Crook et al. demonstrated systemic recurrence is correlated with elevated PSA nadir, as well as reduced PSADT; an average PSA nadir of $0.4 \mathrm{ng} / \mathrm{mL}$ in cases without disease recurrence was reached at 33 months, while $3.2,7.7$, and $1.4 \mathrm{ng} / \mathrm{mL}$ were obtained in individuals with local recurrence at 17 months, distant recurrence at 12 months, and biochemical recurrence at 24 months, respectively [23].

In recent years, high-dose-rate brachytherapy (HDRBT) has attracted increasing attention and is used for more patients. Several clinical trials reported its excellent effects in high-risk patients. Ten-year actuarial biochemical control rates of $100,91,88$, and $79 \%$ were found in subjects with low-, two intermediate-, only one high-, and 2-3 high-risk criteria, respectively $(P=0.004)$; hormone treatment did not affect these results [24]. A 5 -year BRFS of $93.6 \%$ was reported in high-risk patients who underwent ADT + EBRT + HDR-BT, with $87.6 \%$ in the EBRT + HDR-BT group [25]. The overall 3-year OS and BRFS rates were 93.7 and $96.9 \%$ in high-risk cases administered ADT + EBRT + HDR-BT, respectively [26]. Satoshi et al. reported that both LDR-BT + EBRT and HDR-BT + EBRT are safe and suitable for individuals with localized prostate carcinoma, with some advantages of HDR-BT + EBRT over LDR-BT + EBRT in terms of recovery time [27]. Although no further long-term survival data were reported for these two radiation modalities in localized, high-risk patients, LDR-BT is generally administered as a monotherapy in early diagnosed cases, while HDR-BT is usually applied along with EBRT in cases of prostate cancer in unspecified stages [28]. In addition, some clinical trials found that a PSA nadir of less than $0.02 \mathrm{ng} / \mathrm{mL}$ within 12 months of radiotherapy is associated with significantly improved biochemical tumor control and cause-specific survival in cases of locally advanced and non-metastatic high-risk prostate cancer co-administered HDR-BT, EBRT, and longterm ADT [29]. Thorsten et al. reported a discrepant conclusion regarding the predictive value of PSA for the biochemical control rate in 79 cases with highrisk PCa administered HDR-BT following EBRT, with an average follow-up of 21 months; the authors described PSA as a negative predictive biomarker for local recurrence during follow-up, indicating that prolonged follow-up is required for reassessing longterm outcomes [30].

\section{Conclusion}

Overall, brachytherapy is a promising and effective radiation technique, with higher concentration of the radiation dose within the prostate, which decreases the risk of complications in other organs and reduces the frequency of urinary symptoms. PPB-based combined radiotherapy plays an extremely important role in improving OS and BRFS in high-risk PCa patients; time to the first SRE and CCT were also relatively prolonged. These clinical data further demonstrate that post-radiation PSA kinetics could significantly predict survival outcomes in cases with localized, high-risk disease; specifically, PSA nadir $\leq 1 \mathrm{ng} / \mathrm{mL}$, time to PSA nadir $\leq 3$ months, PSA doubling time > 12 months, and PSA reduction $\geq 90 \%$ were associated with improved tumor control. Therefore, more aggressive treatments should be considered for cases with non-favorable PSA kinetics.

\section{Abbreviations}

ADT: Androgen deprivation therapy; BRFS: Biochemical recurrence-free survival; CCT: Cytotoxic chemotherapy; CCT-FS: Cytotoxic chemotherapy-free survival; Cl: Confidence interval; CSS: Cause-specific survival; DE-

EBRT: Dose-escalated external beam radiation therapy; HDR-BT: High-doserate brachytherapy; LDR-BT: Low-dose-rate brachytherapy; LDR-PPB: Lowdose-rate permanent prostate brachytherapy; MAB: Maximal androgen blockade; OS: Overall survival; PCa: Prostate cancer; PSA: Prostate-specific antigen; PSADT: PSA doubling time; RP: Radical prostatectomy; RT: Radiation therapy; SRE: Skeletal-related event; SRE-FS: Skeletal-related event-free survival

\section{Funding}

This work was supported by the National Natural Science Foundation of China (No. 30700968) and High Level Backbone Doctor Training Project of Beijing Health System (No. 2015-3-054). The funders had no role in the study design, data collection and analysis, decision to publish, or preparation of the manuscript. The authors alone are responsible for the content and writing of the paper.

\section{Availability of data and materials}

The details of this procedure are described in the "Methods" section. Please contact the corresponding author for data request.

\section{Authors' contributions}

YLuo and YJ contribute to the conception and design. YLuo, ML, HQ, JZ, YH, YLin, $Z \mathrm{H}$, and $\mathrm{YJ}$ contributed to the development of methodology. YLuo, ML, $H Q, J Z, Y H, Y L i n, Z H$, and YJ contributed to the acquisition of data. YLuo, HQ, and YJ contributed to the analysis and interpretation of data. YLuo, ML, YLin, $\mathrm{ZH}$, and $\mathrm{YJ}$ contributed to the writing, review, and/or revision of the 
manuscript. YLuo and YJ contributed to the supervision of the study. All authors read and approved the final manuscript.

\section{Ethics approval and consent to participate}

The study has been approved by the Committee on the Ethics of Clinical Experiments of the Capital Medical University and has therefore been performed in accordance with the ethical standards laid down in the 1964 Declaration of Helsinki and its later amendments.

\section{Consent for publication}

Written informed consent was obtained from all individuals. Details that might disclose the identity of the subjects under study have been omitted.

\section{Competing interests}

The authors declare that they have no competing interests.

\section{Publisher's Note}

Springer Nature remains neutral with regard to jurisdictional claims in published maps and institutional affiliations.

\section{Received: 28 December 2017 Accepted: 7 May 2018}

\section{Published online: 11 June 2018}

\section{References}

1. Grimm P, Billiet I, Bostwick DG, Dicker AP, Frank S, Immerzeel J, et al. Comparative analysis of prostate-specific antigen free survival outcomes for patients with low, intermediate and high risk prostate cancer treatment by radical therapy. Results from the prostate cancer results study group. BJU Int. 2012;109:22-9.

2. Zelefsky MJ, Reuter VE, Fuks Z, Scardino P, Shippy A. Influence of local tumor control on distant metastases and cancer related mortality after external beam radiotherapy for prostate cancer. J Urol. 2008;179:1368-73.

3. Martinez AA, Gonzalez J, Ye H, Ghilezan M, Shetty S, Kernen K, et al. Dose escalation improves cancer-related events at 10 years for intermediate- and high-risk prostate cancer patients treated with hypofractionated high-doserate boost and external beam radiotherapy. Int J Radiat Oncol Biol Phys. 2011:79:363-70.

4. Sylvester JE, Grimm PD, Wong J, Galbreath RW, Merrick G, Blasko JC. Fifteenyear biochemical relapse-free survival, cause-specific survival, and overall survival following i(125) prostate brachytherapy in clinically localized prostate cancer: Seattle experience. Int J Radiat Oncol Biol Phys. 2011;81:376-81.

5. Davis BJ, Horwitz EM, Lee WR, Crook JM, Stock RG, Merrick GS, et al. American brachytherapy society consensus guidelines for transrectal ultrasound-guided permanent prostate brachytherapy. Brachytherapy. 2012;11:6-19.

6. Pound CR, Partin AW, Eisenberger MA, Chan DW, Pearson JD, Walsh PC Natural history of progression after PSA elevation following radical prostatectomy. JAMA. 1999:281:1591-7.

7. Scher HI, Halabi S, Tannock I, Morris M, Sternberg CN, Carducci MA, et al. Design and end points of clinical trials for patients with progressive prostate cancer and castrate levels of testosterone: recommendations of the Prostate Cancer Clinical Trials Working Group. J Clin Oncol. 2008:26:1148-59.

8. D'Amico AV, Whittington R, Malkowicz SB, Schultz D, Blank K, Broderick GA, et al. Biochemical outcome after radical prostatectomy, external beam radiation therapy, or interstitial radiation therapy for clinically localized prostate cancer. JAMA. 1998;280:969-74.

9. Polascik TJ, Pound CR, DeWeese TL, Walsh PC. Comparison of radica prostatectomy and iodine 125 interstitial radiotherapy for the treatment of clinically localized prostate cancer: a 7-year biochemical (PSA) progression analysis. Urology. 1998;51:884-90.

10. Ragde H, Blasko JC, Grimm PD, Kenny GM, Sylvester JE, Hoak DC, et al. Interstitial iodine-125 radiation without adjuvant therapy in the treatment of clinically localized prostate carcinoma. Cancer. 1997;80:442-53.

11. Sharkey J, Cantor A, Solc Z, Huff W, Chovnick SD, Behar RJ, et al. 103Pd brachytherapy versus radical prostatectomy in patients with clinically localized prostate cancer: a 12-year experience from a single group practice. Brachytherapy. 2005;4:34-44.

12. Taira AV, Merrick GS, Galbreath RW, Butler WM, Lief JH, Adamovich E, et al. Long-term outcomes of prostate cancer patients with Gleason pattern 5 treated with combined brachytherapy and external beam radiotherapy. Brachytherapy. 2013;12:408-14.
13. Demanes DJ, Rodriguez RR, Schour L, Brandt D, Altieri G. High-dose-rate intensity-modulated brachytherapy with external beam radiotherapy for prostate cancer: California endocurietherapy's 10-year results. Int J Radiat Oncol Biol Phys. 2005:61:1306-16.

14. Jani AB, Feinstein JM, Pasciak R, Krengel S, Weichselbaum RR. Role of external beam radiotherapy with low-dose-rate brachytherapy in treatment of prostate cancer. Urology. 2006;67:1007-11.

15. Matzkin H, Keren-Paz G, Mabjeesh NJ, Chen J. Combination therapypermanent interstitial brachytherapy and external beam radiotherapy for patients with localized prostate cancer. Acta Chir lugosl. 2005;52:31-6.

16. Morris WJ, Tyldesley S, Rodda S, Halperin R, Pai H, McKenzie M, et al. Androgen suppression combined with elective nodal and dose escalated radiation therapy (the ASCENDE-RT trial): an analysis of survival endpoints for a randomized trial comparing a low-dose-rate brachytherapy boost to a dose-escalated external beam boost for high- and intermediate-risk prostate cancer. Int J Radiat Oncol Biol Phys. 2017;98:275-85.

17. Antonarakis ES, Zahurak ML, Lin J, Keizman D, Carducci MA, Eisenberger MA Changes in PSA kinetics predict metastasis-free survival in men with PSArecurrent prostate cancer treated with nonhormonal agents: combined analysis of 4 phase II trials. Cancer. 2012;118:1533-42.

18. Crook J, Gillan C, Yeung I, Austen L, McLean M, Lockwood G. PSA kinetics and PSA bounce following permanent seed prostate brachytherapy. Int J Radiat Oncol Biol Phys. 2007;69:426-33.

19. Trapasso JG, de Kernion JB, Smith RB, Dorey F. The incidence and significance of detectable levels of serum prostate specific antigen after radical prostatectomy. J Urol. 1994;152:1821-5.

20. Stewart AJ, Scher HI, Chen MH, McLeod DG, Carroll PR, Moul JW, et al. Prostatespecific antigen nadir and cancer-specific mortality following hormonal therapy for prostate-specific antigen failure. J Clin Oncol. 2005;23:6556-60.

21. D'Amico AV, Chen MH, Roehl KA, Catalona WJ. Identifying patients at risk for significant versus clinically insignificant postoperative prostate-specific antigen failure. J Clin Oncol. 2005;23:4975-9.

22. D'Amico AV, Moul JW, Carroll PR, Sun L, Lubeck D, Chen MH. Surrogate end point for prostate cancer-specific mortality after radical prostatectomy or radiation therapy. J Natl Cancer Inst. 2003:95:1376-83.

23. Crook JM, Choan E, Perry GA, Robertson S, Esche BA. Serum prostate-specific antigen profile following radiotherapy for prostate cancer: implications for patterns of failure and definition of cure. Urology. 1998;51:566-72.

24. Prada PJ, González H, Fernández J, Jiménez I, Iglesias A, Romo I. Biochemical outcome after high-dose-rate intensity modulated brachytherapy with external beam radiotherapy: 12 years of experience. BJU Int. 2012;109: 1787-93

25. Makino T, Mizokami A, Namiki M. Clinical outcomes of patients with localized and locally advanced prostate cancer undergoing high-dose-rate brachytherapy with external-beam radiotherapy at our institute. Anticancer Res. 2015:35:1723-8.

26. Liu J, Kaidu M, Sasamoto R, Ayukawa F, Yamana N, Sato H, et al. Twofraction high-dose-rate brachytherapy within a single day combined with external beam radiotherapy for prostate cancer: single institute experience and outcome. J Radiat Res. 2016:57:280-7.

27. Nakamura S, Murakami N, Inaba K, Wakita A, Kobayashi K, Takahashi K, et al. After low and high dose-rate interstitial brachytherapy followed by IMRT radiotherapy for intermediate and high risk prostate cancer. BMC Cancer. 2016;16:296.

28. Skowronek J. Low-dose-rate or high-dose-rate brachytherapy in treatment of prostate cancer-between options. J Contemp Brachytherapy. 2013;5:33-41.

29. Tsumura H, Satoh T, Ishiyama H, Tabata K, Komori S, Sekiguchi A, et al. Prostate-specific antigen nadir after high-dose-rate brachytherapy predicts long-term survival outcomes in high-risk prostate cancer. J Contemp Brachytherapy. 2016;8:95-103.

30. Ecke TH, Huang-Tiel HJ, Golka K, Selinski S, Geis BC, Koswig S, et al. Prostate specific antigen (PSA) as predicting marker for clinical outcome and evaluation of early toxicity rate after high-dose rate brachytherapy (HDR-BT) in combination with additional external beam radiation therapy (EBRT) for high risk prostate cancer. Int J Mol Sci. 2016;17:E1879. 